\title{
Error Bounds for Compound Quadrature of Weakly Singular Integrals
}

\author{
By Alan Feldstein* and Richard K. Miller**
}

\begin{abstract}
This paper studies the convergence of numerical quadratures of singular integrands. The singularities are ignored in the sense that whenever a singularity occurs the integrand is redefined to be zero. Several convergence theorems are proved under the assumption that the integrand can be dominated near each singularity by a monotone, integrable function.
\end{abstract}

1. Introduction. The primary purpose of this paper is to develop and analyze some practical numerical methods for handling weakly singular quadrature, that is, for $\int_{I} f(t) d t$ where $f$ is Lebesgue integrable on $I$ (so-called "improper integrals"). We also extend this development and analysis to the case where some derivative of $f$ is Lebesgue integrable and has finitely many unbounded points on $I$. We shall be particularly interested in obtaining "best" possible order estimates for compound quadratures.

It is known (although possibly not well known) that Peano's theorem can be applied to analyze the error in "low continuity" numerical quadrature, for example, $\int_{0}^{T} t^{1 / 2} d t$ approximated by the trapezoidal rule:

Let $h>0, N h=T, E(T)=$ error. Then

$$
E(T)=\int_{0}^{T} t^{1 / 2} d t-h\left\{\sqrt{ } h+\sqrt{ } 2 h+\cdots+\sqrt{ }(N-1) h+\frac{1}{2} \sqrt{ } N h\right\} .
$$

By Peano's theorem, cf. e.g. Sard [1, p. 14],

$$
E(T)=\int_{0}^{T} \frac{1}{2} t^{-1 / 2} K(t) d t
$$

where $K(t)=\left(j+\frac{1}{2}\right) h-t$ on $j h \leqq t<(j+1) h$. Apply the Hölder inequality with $1 / p+1 / q=1$ and $1 \leqq q<2$ :

$$
|E(T)| \leqq \frac{1}{2}\left\{\int_{0}^{T}\left|t^{-1 / 2}\right|^{a} d t\right\}^{1 / a}\left\{\int_{0}^{T}|K(t)|^{p} d t\right\}^{1 / p}
$$

Received May 29, 1970, revised January 6, 1971.

AMS 1969 subject classifications. Primary 6555.

Key words and phrases. Quadrature, singular integrals, ignoring the singularity, avoiding the singularity, error bounds, convolution integrals, weakly singular, order of convergence.

* This work was performed under the auspices of the United States Atomic Energy Commission.

** This research was supported in part by the National Aeronautics and Space Administration under Grant No. NGL 40-002-015 and in part by the Air Force Office of Scientific Research under Grant No. AF-AFOSR 67-0693A. 
(Clearly, $\left|t^{-1 / 2}\right|^{a}$ is integrable for $1 \leqq q<2$.) Now, use the definition of $K(t)$ :

$$
\begin{aligned}
\int_{0}^{T}|K(t)|^{p} d t & =N \int_{0}^{h}|h / 2-t|^{p} d t=2 N \int_{0}^{h / 2} t^{p} d t \\
& =(2 N /(p+1))(h / 2)^{p+1}=(h N /(p+1))(h / 2)^{p} \\
& =\{T /(p+1)\}(h / 2)^{p} .
\end{aligned}
$$

Therefore, $\|K\|_{p}=\{T /(p+1)\}^{1 / p}(h / 2)$. Since

$$
\int_{0}^{T}\left|t^{-1 / 2}\right|^{q} d t=\{2 /(2-q)\} T^{1-q / 2},
$$

then

$$
\begin{aligned}
|E(T)| & \leqq(h / 4)\{T /(p+1)\}^{1 / p}\{2 /(2-q)\}^{1 / a} T^{1 / a-1 / 2} \\
& =T^{1 / 2}(p+1)^{-1 / p}\{2 /(2-q)\}^{1 / q}(h / 4) .
\end{aligned}
$$

One could proceed further and analyze the constant

$$
k(q)=(p+1)^{-1 / p}\{2 /(2-q)\}^{1 / a},
$$

subject to $1 / p+1 / q=1,1 \leqq q<2$. One can conclude (by very tedious manipulations) that $k(q)$, for $1 \leqq q<2$, takes on its minimum at $q=1$. In this case, $k(1)=2$. Hence, the minimum estimate on $|E(T)|$ by this application of the Hölder inequality to Peano's theorem is

$$
|E(T)| \leqq(\sqrt{ } T h) / 2 \quad(h=T / N) .
$$

Although this estimate is optimal (in the above sense), it is overly pessimistic, because one can show that $E(T)=\mathcal{O}\left(h^{3 / 2}\right)$. This can be seen in Example 2 in Section 3 below. Indeed, with a little extra care, one can sharpen the result in Example 2 to show that

$$
\frac{1}{6} \leqq h^{-3 / 2} E(T) \leqq \frac{1}{6}+\frac{1}{16} \quad(h=T / N) .
$$

This then clearly demonstrates that applying the Hölder inequality to Peano's theorem may possibly yield substantially less information than is desirable.

In Section 2, we shall show how Peano's theorem and the Hölder inequality can be applied in general to singular quadrature questions. In particular, we generalize the first type of analysis presented above for $\int_{0}^{T} t^{1 / 2} d t$ where $E(T)=\vartheta(h)$. In Section 3, we shall refine our analysis to obtain better information. In particular, we generalize the second type of analysis alluded to above for the case $\int_{0}^{T} t^{1 / 2} d t$ where $E(T)=\mathcal{O}\left(h^{3 / 2}\right)$.

The drawbacks for the usual Hölder-Peano approach become even more exaggerated for $\int_{0}^{T} t^{-1 / 2} d t$ where the integrand itself has a weak singularity. Since the hypothesis of Peano's theorem requires absolute continuity and since $f(t)=t^{-1 / 2}$ is not even continuous at zero, Peano's theorem cannot be applied directly. In Section 4, we show how this situation can be remedied. We propose a simple modification of the usual compound quadrature rule which we call the method of "avoiding the singularity." We then establish general error bounds along with convergence rates for this numerical quadrature of weakly singular integrands.

The use of Peano's theorem to obtain error estimates for quadrature of functions with low continuity is known. For example, Stroud [2] has recently studied certain 
aspects of this method. Numerical quadrature of singular functions has also been studied. Davis and Rabinowitz [3] establish various convergence theorems with interesting lim inf results which were extended by Rabinowitz [4]. Gautschi [5] applied some of the work of Rabinowitz and obtained convergence theorems for two quadratures of interpolatory type. In none of these papers are error bounds explicitly given, although, for example, the proofs in [2] may be used to obtain certain estimates (see [2, line (3.7)], and the proof of Theorem 3). All of these results require that the integrand be monotone in a neighborhood of the singularity. Fox [6] gives some error bounds for singular quadratures. His work is very special and does not seem to generalize.

The main results of this paper predict rather slow convergence rates for weakly singular numerical quadratures. Various numerical experiments verify these predictions. Moreover, there may be no advantage in using a better rule (e.g. Simpson rather than trapezoid); see [7, p. 77] for a striking example of this. If one knows enough about the integrand, it may be possible to change variables or otherwise to eliminate the singularity; see for example [7, pp. 72-73] or [8, pp. 346-352]. In other cases, one might wish to use special numerical quadrature methods which are specifically designed for particular singular integrands. Two examples of such methods are given in Atkinson [9, Sections 2.1 and 2.2] and Schweikert [10].

In Section 5, we apply our results of the earlier sections to the question of singular quadrature in the convolution case. This work in particular will be used in its full generality by the authors in a sequel paper which studies numerical solution of weakly singular Volterra integral equations of the form

$$
x(t)=f(t)+\int_{0}^{t} a(t-s) G(x(s)) d s \quad(0 \leqq t \leqq T)
$$

where $f$ and $G$ are smooth but $a(t)$ may be singular at $t=0 ;\left(a(t)=t^{-1 / 2}\right)$.

2. Basic Estimates. Consider an approximate quadrature rule defined on the standard interval, $0 \leqq t \leqq 1$ :

$$
R(f)=\sum_{i=0}^{J} w_{i} f\left(x_{i}\right)
$$

with error

$$
E(f, R)=\int_{0}^{1} f(t) d t-R(f)
$$

It will always be assumed that the abscissas $x_{i}$ satisfy the inequalities $0 \leqq$ $x_{0}<x_{1}<\cdots<x_{J} \leqq 1$. In addition, we shall assume some or all of the following hypotheses in the sequel:

(A1) $f \in C^{n-1}[0,1]$ where $n \geqq 1$ is a fixed integer and $f^{(n-1)}$ is absolutely continuous on $0 \leqq t \leqq 1$.

(A2) $E(p, R)=0$ for all polynomials $p(t)$ of degree $\leqq n-1$.

(A3) The weights $w_{i}$ are positive for $j=0(1) J$.

(A4) $f \in C^{n-1}[0, T]$ and $f^{(n-1)}$ is absolutely continuous on $[0, T]$.

The symbol $j=0(1) J$ means $j=0,1,2, \cdots, J$. The integer $n \geqq 1$ in hypotheses (A1) and (A2) has the same fixed value. 
For any subinterval $I$ of the real line, let $\chi_{I}(t)$ denote the characteristic function of the interval $I$, that is

$$
\begin{array}{rlll}
\chi_{I}(t)=1 & \text { if } & t \in I, \\
=0 & \text { if } & t \notin I .
\end{array}
$$

For our purposes, the following special case of Peano's theorem will suffice; cf. $[1$, p. 14].

THEOREM 1. Assume the rule (R) together with hypotheses (A1) and (A2). Define

$$
f_{s}(t)=(t-s)^{n-1} \chi_{[0,8]}(t) /(n-1) !
$$

for $0 \leqq s, t \leqq 1$ where $n$ is the integer given in (A1)-(A2). Then the error $E(f, R)$ may be written in the form

$$
E(f, R)=\int_{0}^{1} f^{(n)}(s) K_{n}(s) d s
$$

where $K_{n}(s)=-E\left(f_{s}, R\right)$ for $0 \leqq s \leqq 1$.

The function $K_{n}(s)$ can be explicitly calculated when $R$ and $n$ are known. For example, if $R$ is the midpoint rule, then $J=0, w_{0}=1$ and $x_{0}=\frac{1}{2}$. For $n=1$,

$$
\begin{aligned}
K_{1}(s) & =-s & & \text { if } 0 \leqq s<\frac{1}{2}, \\
& =1-s & & \text { if } \frac{1}{2} \leqq s<1 .
\end{aligned}
$$

In the general case,

$$
K_{n}(s)=(-1)^{n} s^{n} / n !+\sum_{i=0}^{k} w_{i}\left(x_{i}-s\right)^{n-1} /(n-1) !
$$

when $x_{k} \leqq s<x_{k+1}$. If $x_{0}>0$ or if $x_{J}<1$, then similar formulas may be obtained for the intervals $0 \leqq s<x_{0}$ and $x_{J} \leqq s<1$. In particular, (2) shows that $K_{n}$ is of class $L^{p}(0,1)$ for all numbers $p$ in the interval $1 \leqq p \leqq \infty$. Therefore, Theorem 1 and the Hölder inequality imply the following result.

COROLlary 1. Assume the hypotheses of Theorem 1. If $f^{(n)} \in L^{a}(0,1)$ and if $1 / p+1 / q=1$, then

$$
|E(f, R)| \leqq\left\{\int_{0}^{1}\left|K_{n}(s)\right|^{p} d s\right\}^{1 / p}\left\{\int_{0}^{1}\left|f^{(n)}(s)\right|^{a} d s\right\}^{1 / a} \quad(1<q<\infty)
$$

with similiar formulas for the cases $q=1$ and $q=\infty$.

In order to simplify the notation, introduce the $L^{p}$ norms

$$
\|f\|_{p(A, B)}=\left\{\int_{A}^{B}|f(t)|^{p} d t\right\}^{1 / p} \quad(1 \leqq p<\infty)
$$

and a similar norm when $p=+\infty$. When $(A, B)$ is the standard interval $(0,1)$, then the interval designation will be dropped. Thus, one has

$$
\left\|K_{n}\right\|_{p}=\left\{\int_{0}^{1}\left|K_{n}(t)\right|^{p} d t\right\}^{1 / p} \quad(1 \leqq p<\infty)
$$

and

$$
\left\|K_{n}\right\|_{\infty}=\max \left\{\left|K_{n}(s)\right|: 0 \leqq s \leqq 1\right\}
$$


In this notation, (3) reads

$$
|E(f, R)| \leqq\left\|K_{n}\right\|_{p}\left\|f^{(n)}\right\|_{a} .
$$

In general, the calculations of the $L^{p}$ norms of $K_{n}$ may be difficult if $1 \leqq p<\infty$. Since the number $\left\|K_{n}\right\|_{\infty}$ is easy to obtain numerically, then it may be convenient to use the estimate $\left\|K_{n}\right\|_{p} \leqq\left\|K_{n}\right\|_{\infty}$. If this is not sufficient, then it is possible to obtain a universal estimate under the additional hypothesis (A3).

COROLlaRY $1^{*}$. Assume the hypotheses of Theorem 1. If $f^{(n)} \in L^{a}(0,1)$ and $1 / p+1 / q=1$, then

$$
|E(f, R)| \leqq\left\|K_{n}\right\|_{p}\left\|f^{(n)}\right\|_{a} \leqq\left\|K_{n}\right\|_{\infty}\left\|f^{(n)}\right\|_{a} .
$$

If in addition (A3) is true, then $\left\|K_{n}\right\|_{\infty} \leqq(n+1) / n$ ! .

Proof. Only the last statement needs further proof. Assumptions (A2) and (A3) imply that $\sum_{j=0}^{J} w_{j}=1$. For $k=0(1) J$ and for $s$ in the interval $x_{k} \leqq s<x_{k+1}$, Eq. (2) implies that

$$
\begin{aligned}
\left|K_{n}(s)\right| & \leqq 1 / n !+\sum_{i=0}^{k} w_{i} /(n-1) ! \\
& \leqq 1 / n !+\left(\sum_{j=0}^{J} w_{i}\right) /(n-1) !=(n+1) / n !
\end{aligned}
$$

If $x_{0}>0$, then $\left|K_{n}(s)\right| \leqq 1 / n ! \leqq(n+1) / n !$ in the interval $0 \leqq s<x_{0}$. Similarly, if $x_{J}<1$ and if $x_{J} \leqq s<1$, then $\left|K_{n}(s)\right| \leqq(n+1) / n$ !. Q.E.D.

These basic results are easily extended to general intervals and to compound rules. Given an interval $a \leqq t \leqq a+B$, one can use the transformation $\tau=a+B t$ to compute

$$
\int_{a}^{a+B} F(\tau) d \tau=B \int_{0}^{1} F(a+B t) d t \doteq B \sum_{i=0}^{J} w_{i} F\left(a+B x_{i}\right) .
$$

For example, if $(R)$ is the midpoint rule $R(f)=f\left(\frac{1}{2}\right)$, then one can replace $B$ by $h$ and write

$$
\int_{a}^{a+h} F(\tau) d \tau \doteq h F(a+h / 2)
$$

in the usual way. If $(R)$ is Simpson's rule, then we think of $B=2 h$ and write

$$
\int_{a}^{a+2 h} F(\tau) d \tau \doteq(h / 3)\{F(a)+4 F(a+h)+F(a+2 h)\} .
$$

Corollary 2. Assume (A2) is true, $F \in C^{n-1}[a, a+B]$ and $F^{(n-1)}$ is absolutely continuous on $a \leqq t \leqq a+B$. Then all the following statements are true.

a. The error $E=\int_{a}^{a+B} F(t) d t-B \sum_{i=0}^{J} w_{i} F\left(a+B x_{i}\right)$ may be written in the form

$$
E=B^{n} \int_{a}^{a+B} K_{n}\left(\frac{s-a}{B}\right) F^{(n)}(s) d s
$$

where $K_{n}(s)$ is the function given in Theorem 1 above.

b. If $F^{(n)} \in L^{a}(a, a+B)$, if $1 / p+1 / q=1$ and if $\left\|K_{n}\right\|_{p}$ is the $L^{p}$ norm of $K_{n}$ over the interval $[0,1]$ (see (4.1)), then

$$
|E| \leqq B^{n+1 / p}\left\|K_{n}\right\|_{D}\left\{\int_{a}^{a+B}\left|F^{(n)}(s)\right|^{a} d s\right\}^{1 / a} .
$$


c. If, in addition to the hypotheses listed above, (A3) is also true, then

$$
|E| \leqq B^{n+1 / p}(n+1) / n !\left\{\int_{a}^{a+B}\left|F^{(n)}(s)\right|^{a} d s\right\}^{1 / a} \text {. }
$$

Proof. By Theorem 1 above

$$
\begin{aligned}
E & =B \int_{0}^{1} F(a+B t) d t-B \sum_{i=0}^{J} w_{j} F\left(a+B x_{i}\right) \\
& =B \int_{0}^{1} K_{n}(s)\left\{d^{n} F(a+B s) / d s^{n}\right\} d s \\
& =B^{n+1} \int_{0}^{1} K_{n}(s) F^{(n)}(a+B s) d s \\
& =B^{n} \int_{a}^{a+B} K_{n}\left(\frac{s-a}{B}\right) F^{(n)}(s) d s .
\end{aligned}
$$

This proves $\mathrm{a}$. Part $\mathrm{b}$ follows from part $\mathrm{a}$ and $\mathrm{a}$ change of variables:

$$
\left\{\int_{a}^{a+B}\left|K_{n}\left(\frac{s-a}{B}\right)\right|^{p} d s\right\}^{1 / p}=\left\{\int_{0}^{1}\left|K_{n}(s)\right|^{p} B d s\right\}^{1 / p}=B^{1 / p}\left\|K_{n}\right\|_{p} .
$$

Part c follows from $\mathrm{b}$ since $\left\|K_{n}\right\|_{p} \leqq\left\|K_{n}\right\|_{\infty} \leqq(n+1) / n$ !. Q.E.D.

Now, consider a compounding of the rule $R$ over an interval $0 \leqq t \leqq T$. Let $T=N B$ where $B>0$ and where $N$ is an integer larger than one. If $R$ is compounded $N$ times over $[0, T]$, then

$$
(N \times R) \quad N \times R(f)=\sum_{k=0}^{N-1}\left\{\sum_{i=0}^{J} B w_{i} f\left(B x_{i}+k B\right)\right\} .
$$

Let $E_{N}(f)=\int_{0}^{T} f(t) d t-N \times R(f)$ be the error.

COROLlARY 3. Suppose (A2) and (A4) are true. Then all of the following statements are true:

a. $E_{N}(f)=\int_{0}^{T} f^{(n)}(t) \bar{K}_{n}(t) d t$ where $\bar{K}_{n}(t)=K_{n}(t / B-k)$ on $k B \leqq t<(k+1) B$.

b. If $f^{(n)} \in L^{a}(0, T), 1 / p+1 / q=1$ and $\left\|K_{n}\right\|_{p}$ is defined as in (4.1)-(4.2), then

$$
\left|E_{N}(f)\right| \leqq B^{n} T^{1 / p}\left\|K_{n}\right\|_{p}\left\|f^{(n)}\right\|_{a(0, T)} .
$$

c. If in addition (A3) is true, then

$$
\left|E_{N}(f)\right| \leqq\left\{B^{n} T^{1 / p}(n+1) / n !\right\}\left\|f^{(n)}\right\|_{Q(0, T)} .
$$

Proof. Write the error in the form

$$
E_{N}(f)=\sum_{k=0}^{N-1}\left\{\int_{k B}^{k B+B} f(t) d t-\sum_{j=0}^{J} B w_{i} f\left(B\left(x_{i}+k\right)\right)\right\},
$$

and then apply Corollary 2a:

$$
E_{N}(f)=\sum_{k=0}^{N-1} B^{n} \int_{k B}^{k B+B} f^{(n)}(t) K_{n}(t / B-k) d t=B^{n} \int_{0}^{T} f^{(n)}(t) \bar{K}_{n}(t) d t
$$


Since $\bar{K}_{n}(t)$ is periodic of period $B$ on $0 \leqq t \leqq T$, then

$$
\begin{aligned}
\int_{0}^{T}\left|\bar{K}_{n}(t)\right|^{p} d t & =N \int_{0}^{B}\left|\bar{K}_{n}(t)\right|^{p} d t=N B \int_{0}^{1}\left|K_{n}(t)\right|^{p} d t \\
& \left.=T\left\{\left\|K_{n}\right\|\right\}_{p}\right\}^{p} .
\end{aligned}
$$

Therefore,

$$
\begin{aligned}
B^{-n}\left|E_{N}(f)\right| & \leqq\left\{\int_{0}^{T}\left|f^{(n)}(t)\right|^{Q} d t\right\}^{1 / Q}\left\{\int_{0}^{T}\left|K_{n}(t)\right|^{p} d t\right\}^{1 / p} \\
& =\left\|f^{(n)}\right\|_{Q(0, T)} T^{1 / p}|| K_{n} \|_{p} .
\end{aligned}
$$

If (A3) is true, then $\left\|K_{n}\right\|_{p} \leqq\left\|K_{n}\right\|_{\infty} \leqq(n+1) / n$ !. Q.E.D.

Example 1. Consider $\int_{0}^{1} t^{1 / 2} d t$ approximated by the trapezoidal rule. In this case, $B=h, T=N h=1, n=1$ and $K_{1}(s)=\frac{1}{2}-s$ on the interval $0 \leqq s<1$. Therefore,

$$
\begin{aligned}
\int_{0}^{1}\left|K_{1}(s)\right|^{p} d s & =\int_{0}^{1}\left|\frac{1}{2}-s\right|^{p} d s=\int_{0}^{1 / 2}\left|\frac{1}{2}-s\right|^{p} d s+\int_{1 / 2}^{1}\left|\frac{1}{2}-s\right|^{p} d s \\
& =\int_{0}^{1 / 2} s^{p} d s+\int_{0}^{1 / 2} s^{p} d s=2^{-p}(p+1)^{-1} .
\end{aligned}
$$

Since $f^{\prime}(t)=\left(2 t^{1 / 2}\right)^{-1} \in L^{a}(0,1)$ for $1 \leqq q<2$, then Corollary 3 implies that

$$
\begin{aligned}
\left|E_{N}\left(t^{1 / 2}\right)\right| & \leqq h 2^{-1}(p+1)^{-1 / p}\left\{\int_{0}^{1}\left(2 t^{1 / 2}\right)^{-q} d t\right\}^{1 / q} \\
& =h\left\{2^{1 / q-2}(p+1)^{-1 / p}(2-q)^{-1 / q}\right\}
\end{aligned}
$$

for $1<q<2$. If $q=1$, then

$$
\left|E_{N}\left(t^{1 / 2}\right)\right| \leqq h\left(\frac{1}{2}\right) \int_{0}^{1}\left(2 t^{1 / 2}\right)^{-1} d t=h / 2 .
$$

Part c of Corollary 3 implies an even more pessimistic estimate $\left|E_{N}\left(t^{1 / 2}\right)\right| \leqq 2 h$. Section 3 will produce estimates which are $\theta\left(h^{3 / 2}\right)$.

\section{Refined Estimates.}

Definition 1. A function $f$ is said to be weakly singular of order $\nu$ if and only if

a. $f \in C(0, T]$ if $\nu=0$ or $f \in C^{\nu-1}[0, T] \cap C^{\nu}(0, T]$ if $\nu \geqq 1$,

b. for each $\epsilon>0$ the function $f^{(\nu)}(t)$ is absolutely continuous on the interval $\epsilon \leqq t \leqq T$, and

c. the function $\alpha_{\nu}$ defined by

$$
\alpha_{\nu}(t, f)=\left|f^{(\nu)}(T)\right|+\int_{t}^{T}\left|f^{(\nu+1)}(s)\right| d s
$$

is of class $L^{1}(0, T)$.

For any integer $N \geqq 0$, let WS $(\nu)$ denote the set of all functions $f$ which are weakly singular of order $\nu$. For example, if $0<r<1$ and $T=1$, then $f(t)=\log t$ and $g(t)=\sin \left(t^{-r}\right)$ are in WS(0). In these two cases, $\alpha_{0}(t, f)=-\log t$ and $\alpha_{0}(t, g) \leqq t^{-r}$. In general, $f(t)=t^{\nu-r}, 0<r<1$, is of class WS( $(\nu)$ so that each class WS $(\nu)$ is not empty. 
LEMMA 1. If $f \in \mathrm{WS}(\nu)$, then $\left|f^{(v)}(t)\right| \leqq \alpha_{\nu}(t, f)$ on the interval $0<t \leqq T$. Moreover, $\alpha_{,}(t, f)$ is nonincreasing in $t$.

Proof. Fix $f$ in WS $(\nu)$. The absolute continuity of $f^{(\nu)}$ implies that

$$
f^{(v)}(t)=f^{(v)}(T)-\int_{t}^{T} f^{(v+1)}(s) d s
$$

for any $t$ in the interval $0<t \leqq T$. This formula and (7) imply that

$$
\left|f^{(n)}(t)\right| \leqq\left|f^{(v)}(T)\right|+\int_{t}^{T}\left|f^{(v+1)}(s)\right| d s=\alpha_{v}(t, f)
$$

when $0<t \leqq T$.

From (7) it follows that $\alpha$, is absolutely continuous on $\epsilon \leqq t \leqq T$ and that

$$
\frac{d}{d t} \alpha_{\nu}(t, f)=-\left|f^{(\nu+1)}(t)\right| \leqq 0 \quad \text { a.e. }
$$

Thus, $\alpha$, is nonincreasing on the interval $\epsilon \leqq t \leqq T$. Since $\epsilon>0$ can be made arbitrarily small, the proof is complete. Q.E.D.

THEOREM 2. Suppose (A2) is true for some integer $n=\nu+1$ where $\nu \geqq 1$. If $f \in \mathrm{WS}(\nu)$ then the error $E_{N}(f)$ obtained by applying the compound rule $(N \times R)$ satisfies the inequality

$$
\left|E_{N}(f)\right| \leqq B^{\prime} \int_{0}^{B} \alpha_{\nu}(t, f) d t\left\{\left\|K_{\nu}\right\|_{\infty}+\left\|K_{\nu+1}\right\|_{\infty}\right\}
$$

In particular, if (A3) is also true, then

$$
\left|E_{N}(f)\right| \leqq B^{\nu}\left(\nu^{2}+3 \nu+3\right) /(\nu+1) !\left\{\int_{0}^{B} \alpha_{\nu}(t, f) d t\right\}
$$

Proof. Write $E=E_{N}(f)$ in the form

$$
\begin{aligned}
E= & \left\{\int_{0}^{B} f(t) d t-\sum_{i=0}^{J} B w_{i} f\left(B x_{i}\right)\right\} \\
& +\left\{\int_{0}^{T-B} f(t+B) d t-\sum_{k=0}^{N-2} B w_{i} f\left(B\left(x_{i}+(k+1)\right)\right)\right\}
\end{aligned}
$$

Apply Corollary $3 \mathrm{~b}$ to the first summand with $n=\nu, q=1$, and to the second summand with $n=\nu+1, q=1$ :

$$
|E| \leqq B^{\prime}|| K_{\nu}\left\|_{\infty}\left\{\int_{0}^{B}\left|f^{(\nu)}(t)\right| d t\right\}+B^{\nu+1}|| K_{\nu+1}\right\|_{\infty}\left\{\int_{0}^{T-B}\left|f^{(\nu+1)}(t+B)\right| d t\right\} .
$$

Now use (7) and Lemma 1:

$$
|E| \leqq B^{\prime}\left\|K_{\nu}\right\|_{\infty} \int_{0}^{B} \alpha_{\nu}(t, f) d t+B^{\nu+1}\left\|K_{\nu+1}\right\|_{\infty}\left\{\alpha_{\nu}(B, f)-\alpha_{\nu}(T, f)\right\}
$$

Since $\alpha_{v}(t, f)$ is nonnegative and nonincreasing in $t$, then

$$
B\left\{\alpha_{\nu}(B, f)-\alpha_{\nu}(T, f)\right\} \leqq B \alpha_{\nu}(B, f) \leqq \int_{0}^{B} \alpha_{\nu}(t, f) d t .
$$


Therefore (8) follows. The estimate (9) follows from (8) and the inequality $\left\|K_{n}\right\|_{\infty} \leqq(n+1) / n$ !. Q.E.D.

Example 2. Consider $\int_{0}^{1} t^{1 / 2} d t$ approximated by the trapezoidal rule. (The same example as at the end of Section 2.) Then $B=h, T=1=N h, n=2, \nu=1$ and

$$
K_{1}(s)=\frac{1}{2}-s, \quad K_{2}(s)=s(s-1) / 2 .
$$

It is easy to compute $\alpha_{1}(t)=\frac{1}{2} t^{-1 / 2}$ and $\int_{0}^{h} \alpha_{1}(t) d t=h^{1 / 2}$. Therefore, (8) implies that

$$
\left|E_{N}\left(t^{1 / 2}\right)\right| \leqq h\left\{\frac{1}{2}+\frac{1}{8}\right\} h^{1 / 2}=\frac{5}{8} h^{3 / 2} .
$$

Even less computation is required to see that (9) implies

$$
\left|E_{N}\left(t^{1 / 2}\right)\right| \leqq \frac{7}{2} h^{8 / 2} .
$$

Either result shows that the error is of order $\theta\left(h^{3 / 2}\right)$ as $h=1 / N \rightarrow 0$. The estimates in Section 2 were $\mathcal{O}(h)$.

Theorem 2 above cannot be applied if the integer $n$ in hypothesis (A2) is equal to one but $f \in \mathrm{WS}(\nu)$ for some integer $\nu \geqq 1$. However, such situations are already covered by Corollary 3 above. For example, if the midpoint rule $M$ is applied to $f(t)=t^{1 / 2}$, then, by Corollary 3 , the error is $\mathcal{O}\left(h^{1 / 2}\right)$. The reverse situation $\nu=0$ and $n \geqq 1$ is more complicated. This situation is the topic of the next section.

4. Estimates when $\nu=0$. If $f \in \mathrm{WS}(\nu)$ and $\nu=0$, then $t=0$ may be an unbounded point of $f$. In this case, the compound rule $(N \times R)$ need not be well-defined. Even if $(N \times R)$ is well-defined (e.g. if rule $R$ is open at $t=0)$, the previous estimates do not apply. One simple method of handling both of these problems is to avoid the singularity at $t=0$. This idea leads to the following approximation rule:

Let $T=N B$ where $B>0$ and $N>1$ is an integer. Compute

$$
R_{A}(T, f)=\sum_{k=1}^{N-1}\left\{\sum_{i=0}^{J} B w_{i} f\left(x_{i} B+k B\right)\right\}
$$

and let $E_{A}(f, N)=$ error.

Rule $R_{A}$ will be called the method of "avoiding the singularity." This rule is the usual compound rule except that no attempt is made to approximate on the initial segment $[0, B]$.

THEOREM 3. Suppose (A2) is true for $n=1$. If $f \in \mathrm{WS}(0)$ then

$$
\left|E_{A}(f, N)\right| \leqq\left\{1+\left\|K_{1}\right\|_{\infty}\right\} \int_{0}^{B} \alpha_{0}(t, f) d t .
$$

In particular, if (A3) is also true, then

$$
\left|E_{A}(f, N)\right| \leqq 3 \int_{0}^{B} \alpha_{0}(t, f) d t .
$$

Proof. Define $f_{B}(t)=f(t+B)$ on $0 \leqq t \leqq T-B$. Then the error $E_{A}=E_{A}(f, N)$ has the form

$$
E_{A}=\int_{0}^{B} f(t) d t+E_{N-1}\left(f_{B}\right),
$$


where $E_{k}(g)$ is the error for the usual compound rule. Apply Corollary $3 b$ with $n=q=1$ :

$$
\begin{aligned}
\left|E_{A}\right| & \leqq \int_{0}^{B}|f(t)| d t+B|| K_{1} \|_{\infty} \int_{0}^{T-B}\left|\left(f_{B}\right)^{\prime}(t)\right| d t \\
& =\int_{0}^{B}|f(t)| d t+B\left\|K_{1}\right\|_{\infty} \int_{B}^{T}\left|f^{\prime}(t)\right| d t .
\end{aligned}
$$

Use Lemma 1 and the definition (7):

$$
\int_{0}^{B}|f(t)| d t \leqq \int_{0}^{B} \alpha_{0}(t, f) d t
$$

and

$$
\begin{aligned}
\int_{B}^{T}\left|f^{\prime}(t)\right| d t & =\alpha_{0}(B, f)-\alpha_{0}(T, f) \\
& \leqq(1 / B) B \alpha_{0}(B, f) \leqq(1 / B) \int_{0}^{B} \alpha_{0}(t, f) d t
\end{aligned}
$$

This proves (10).

If (A3) is also true, then $\left\|K_{1}\right\|_{\infty} \leqq(1+1) / 1 !=2$. Therefore, (11) follows immediately from (10). Q.E.D.

Example 3. If $f(t)=t^{-r}, 0<r<1$, then it is easy to compute $\alpha_{0}(t, f)=t^{-r}$. Theorem 3 predicts $E_{A}=\mathcal{O}\left(B^{1-r}\right)$ as $B \rightarrow 0$. If $f(t)=t^{-r} \sin \left(t^{-v}\right)$, where $r, \nu>0$ and $r+\nu<1$, then, by Theorem 3, one has at least $E_{A}=\mathcal{O}\left(B^{1-r-\nu}\right)$. If $f(t)=t^{-r} \sin (\log t)$ where $0<r<1$, then $E_{A}=\mathcal{O}\left(B^{1-r}\right)$, at least.

5. Convolution Integrals. Consider a convolution integral

$$
I=\int_{0}^{T} f(T-s) g(s) d s=\int_{0}^{T} f(s) g(T-s) d s .
$$

THEOREM 4. Suppose (A2) is true for $n=\nu+1$. In addition, assume

i. $f \in \mathrm{WS}(\nu)$ where $\nu \geqq 1$ and

ii. $g \in C^{\nu}[0, T] \cap C^{\nu+1}(0, T]$ with $g^{(\nu+1)} \in L^{1}(0, T)$.

Define $F(t)=f(t) g(T-t)$ on $0 \leqq t \leqq T$. Let $L>0$ be a bound for each of the functions $|f(t)|,|g(t)|,\left|g^{\prime}(t)\right|, \cdots,\left|g^{(v)}(t)\right|$ on the interval $0 \leqq t \leqq T$. Then the error $E_{N}(F)$, obtained by applying the compound rule $N \times R$ to $F$, satisfies the estimate

$$
\begin{aligned}
\left|E_{N}(F)\right| \leqq & L B^{\nu}\left(\left\|K_{\nu}\right\|_{\infty}+\left\|K_{\nu+1}\right\|_{\infty}\right) \\
& \cdot\left\{\int_{0}^{B} \alpha_{\nu}(t, f) d t+B \int_{0}^{T}\left(\left|g^{(\nu+1)}(s)\right|+\sum_{j=1}^{p}\left(\begin{array}{c}
\nu+1 \\
j
\end{array}\right)\left|f^{(j)}(s)\right|\right) d s\right\},
\end{aligned}
$$

where $\left(\begin{array}{l}k \\ j\end{array}\right)=k ! / j !(k-j) !$.

Proof. For any number $s$ in the interval $0<s \leqq T$, one has

$$
F^{(v+1)}(s)=\sum_{i=0}^{p+1}(-1)^{i}\left(\begin{array}{c}
\nu+1 \\
j
\end{array}\right) f^{(\nu+1-i)}(s) g^{(i)}(T-s) .
$$


Therefore, if $L$ is the bound defined above,

$$
\left|F^{(v+1)}(s)\right| \leqq L\left\{\sum_{j=0}^{p}\left(\begin{array}{c}
\nu+1 \\
j
\end{array}\right)\left|f^{(\nu+1-i)}(s)\right|\right\}+L\left|g^{(\nu+1)}(T-s)\right| .
$$

This shows that $F \in \mathrm{WS}(\nu)$ and

$$
\alpha_{\nu}(t, F) \leqq L \alpha_{v}(t, f)+L \int_{t}^{T}\left\{\left|g^{(v+1)}(T-s)\right|+\sum_{j=1}^{j}\left(\begin{array}{c}
\nu+1 \\
j
\end{array}\right)\left|f^{(i)}(s)\right|\right\} d s .
$$

Apply Theorem 2:

$$
\begin{aligned}
\left|E_{N}(F)\right| \leqq B^{\prime}\left(\left\|K_{\nu}\right\|_{\infty}+\left\|K_{p+1}\right\|_{\infty}\right) \int_{0}^{B} \alpha_{\nu}(t, F) d t \\
\leqq L B^{\prime}\left(\left\|K_{\nu}\right\|_{\infty}+\left\|K_{p+1}\right\|_{\infty}\right) \\
\cdot\left\{\int_{0}^{B} \alpha_{\nu}(t, f) d t+\int_{0}^{B} \int_{t}^{T}\left(\left|g^{(p+1)}(T-s)\right|+\sum_{j=1}^{p}\left(\begin{array}{c}
\nu+1 \\
j
\end{array}\right)\left|f^{(j)}(s)\right|\right) d s d t\right\} .
\end{aligned}
$$

By nonnegativity and Fubini's theorem,

$$
\begin{aligned}
\int_{0}^{B} \int_{t}^{T}\left(\left|g^{(p+1)}(T-s)\right|\right. & \left.+\sum_{i=1}^{p}\left(\begin{array}{c}
\nu+1 \\
j
\end{array}\right)\left|f^{(j)}(s)\right|\right) d s d t \\
& \leqq \int_{0}^{B} \int_{0}^{T}(\cdots) d s d t=\int_{0}^{T} \int_{0}^{B}(\cdots) d t d s \\
& =B \int_{0}^{T}\left(\left|g^{(p+1)}(s)\right|+\sum_{j=1}^{j}\left(\begin{array}{c}
\nu+1 \\
j
\end{array}\right)\left|f^{(i)}(s)\right|\right) d s . \quad \text { Q.E.D. }
\end{aligned}
$$

The next set of results follow immediately from Theorem 4.

Corollary 4. Suppose the hypotheses of Theorem 4 are true. Pick any number $M_{1}>0$ which satisfies the estimate

$$
b \leqq M_{1} \int_{0}^{b} \alpha_{\nu}(t, f) d t \quad(0<b \leqq T) .
$$

Then $\left|E_{N}(F)\right| \leqq M B^{\nu} \int_{0}^{B} \alpha_{\nu}(t, f) d t$ where

$$
\begin{aligned}
M= & L\left(\left\|K_{\nu}\right\|_{\infty}+\left\|K_{p+1}\right\|_{\infty}\right) \\
& \cdot\left\{1+M_{1} \int_{0}^{T}\left(\left|g^{(\nu+1)}(s)\right|+\sum_{i=1}^{\infty}\left(\begin{array}{c}
\nu+1 \\
j
\end{array}\right)\left|f^{(i)}(s)\right|\right) d s\right\} .
\end{aligned}
$$

COROLlary 5. Suppose the hypotheses of Theorem 4 are true. For any integer $N>0$ let $B=T / N$ and define

$$
I_{j}=\int_{0}^{j B} f(s) g(j B-s) d s \quad(j=1(1) N) .
$$

Let $E_{j}$ be the error in approximating $I_{j}$ by the compound rule $(j \times R)$. Then

$$
\left|E_{j}\right| \leqq M B^{\nu} \int_{0}^{B} \alpha_{\nu}(t, f) d t \quad(j=1(1) N),
$$

where $M$ is the constant defined by (14). In particular, $M$ is independent of $N \geqq 1$ and of $j=1(1) N$. 
A similar analysis is obtained when $\nu=0$.

THEOREM 5. Suppose (A2) is true for $n=1$. Assume $f \in \mathrm{WS}(0), g$ is absolutely continuous on $0 \leqq t \leqq T$ and $L$ is $a$ bound on $|g(t)|$ for $0 \leqq t \leqq T$. Define

$$
F(s)=f(s) g(T-s) \quad(0<t \leqq T) .
$$

Then, the error $E_{A}$, obtained by applying the method avoiding the singularity to $F$, satisfies the estimate

$$
\left|E_{A}(f, N)\right| \leqq\left(1+\left\|K_{1}\right\|_{\infty}\right)\left(L+\int_{0}^{T}\left|g^{\prime}(s)\right| d s\right) \int_{0}^{B} \alpha_{0}(t, f) d t .
$$

Proof. Since $F^{\prime}(s)=f^{\prime}(s) g(T-s)-f(s) g^{\prime}(T-s)$, then $F \in \mathrm{WS}(0)$ and

$$
\begin{aligned}
\left|F^{\prime}(s)\right| & \leqq L\left|f^{\prime}(s)\right|+|f(s)|\left|g^{\prime}(T-s)\right| \\
& \leqq L\left|f^{\prime}(s)\right|+\alpha_{0}(s, f)\left|g^{\prime}(T-s)\right|
\end{aligned}
$$

on $0<s<T$. In particular, then

$$
\alpha_{0}(t, F) \leqq L \alpha_{0}(t, f)+\int_{t}^{T} \alpha_{0}(s, f)\left|g^{\prime}(T-s)\right| d s .
$$

Apply Theorem 3:

$$
\begin{aligned}
\left|E_{A}\right| & \leqq\left(1+\left\|K_{1}\right\|_{\infty}\right) \int_{0}^{B} \alpha_{0}(t, F) d t \\
& \leqq\left(1+\left\|K_{1}\right\|_{\infty}\right)\left(L \int_{0}^{B} \alpha_{0}(t, f) d t+\int_{0}^{B} \int_{t}^{T} \alpha_{0}(s, f)\left|g^{\prime}(T-s)\right| d s d t\right) .
\end{aligned}
$$

Since $\alpha_{0}(t, f)$ is nonnegative and monotone in $t$, then

$$
\begin{aligned}
\int_{0}^{B} \int_{t}^{T} \alpha_{0}(s, f)\left|g^{\prime}(T-s)\right| d s d t & \leqq \int_{0}^{B} \alpha_{0}(t, f) \int_{t}^{T}\left|g^{\prime}(T-s)\right| d s d t \\
& \leqq\left(\int_{0}^{B} \alpha_{0}(t, f) d t\right)\left(\int_{0}^{T}\left|g^{\prime}(s)\right| d s\right) . \quad \text { Q.E.D. }
\end{aligned}
$$

Corollary 6. Assume the hypotheses of Theorem 5. For any integer $N>0$, let $B=T / N$ and define

$$
I_{i}=\int_{0}^{i B} f(s) g(j B-s) d s \quad(j=1(1) N) .
$$

Let $E_{A}(j)$ be the error obtained in approximating $I_{i}$ by the rule $R_{A}$. Then

$$
\left|E_{j}\right| \leqq\left(1+\left\|K_{1}\right\|_{\infty}\right)\left(L+\int_{0}^{T}\left|g^{\prime}(s)\right| d s\right) \int_{0}^{B} \alpha_{0}(t, f) d t,
$$

where $L$ is the bound defined in Theorem 5 . (This estimate is independent of $N$ and $j$. .)

6. Numerical Examples. The results in Sections 3 and 4 were verified by numerically integrating the function $f(t)=\alpha t^{a} \sin \left(t^{-b}\right)$ for various values of the parameters $\alpha, a$ and $b$ with $-1<a<1$ and $0 \leqq b<1$. These computations were performed on a CDC 6600 computer at the Lawrence Radiation Laboratory, U. S. 
Atomic Energy Commission. They were designed and implemented with the help of Dr. Fred Fritsch.

Some computations were recomputed in double precision. The numerical evidence obtained in this way suggests that round-off errors had no effects on the calculations over the full range of values of $h$. Experiments were made using both the method of "ignoring" the singularity (see [3]) and the method of "avoiding" the singularity (Section 3 above). "Avoiding" is easier to handle theoretically while "ignoring" was a bit easier to program. Ignoring gives slightly better accuracy for monotone integrands while avoiding may be a bit better for oscillating integrands.

Table 1 contains results for the integral

$$
I=(1+a) \int_{0}^{1} t^{a} d t=1,
$$

for the value $a=-\frac{1}{4}$. Simpson's rule was employed with $h=2^{-k}$ and $k=1(1) 15$. The constants $C(h)$ in Table 1 were computed by putting the error in the form $E(h)=C(h) h^{1+a}$. Then

$$
C(h)=E(h) / h^{1+a} .
$$

These values of $C(h)$ appear to be converging as $h \rightarrow 0$. As a second check on the possible asymptotic form of the error, one can assume that $E(h)=C_{0} h^{\rho}$ (at least asymptotically). Then $\rho$ may be calculated using the formulas

$$
Q=\frac{E(h)-E(2 h)}{E(h / 2)-E(h)}, \quad \rho=\frac{\log Q}{\log 2} .
$$

(One can also calculate $C_{0}$ in this manner.) The last column in Table 1 is computed using (17).

TABLE 1. $f(t)=.75 t^{-.25}$

\begin{tabular}{rccc}
\hline$k$ & Error & $C$ & $\rho$ \\
\hline 1 & -.36655 & -.61645 & \\
2 & -.21663 & -.61271 & \\
3 & -.12847 & -.61112 & .76609 \\
4 & -.07631 & -.61045 & .75692 \\
5 & -.04535 & -.61016 & .75293 \\
6 & -.02696 & -.61005 & .75123 \\
7 & -.01629 & -.61000 & .75052 \\
8 & -.00953 & -.60997 & .75022 \\
9 & -.00567 & -.60995 & .75009 \\
10 & -.00337 & -.60996 & .75004 \\
11 & -.00200 & -.60996 & .75002 \\
12 & -.00119 & -.60996 & .75001 \\
13 & -.00071 & -.60996 & .75000 \\
14 & -.00042 & -.60996 & .75000 \\
15 & -.00025 & -.60996 & .75000 \\
\hline
\end{tabular}


Table 1 indicates slow but monotone convergence. The error appears to have the form $E(h)=C(h) h^{3 / 4}$ where $C(h) \rightarrow C_{0}=-.60996 \cdots$ as $h \rightarrow 0$. This general behavior is typical of the integrand (15) for $0<|a|<1$. For example, if $a=.75$ in (15), Simpson's rule gives much more rapid monotone convergence:

$$
S\left(2^{-5}\right)=.99956, \quad S\left(2^{-10}\right)=.9999988, \quad S\left(2^{-15}\right)=.999999997 .
$$

In $E(h)=C(h) h^{1.75}$, the corresponding values of $C(h)$ are:

$$
C\left(2^{-5}\right)=.1878, \quad C\left(2^{-10}\right)=.2145, \quad C\left(2^{-15}\right)=.2265 .
$$

The values of $C(h)$ appear to be converging rather slowly. If $a=-.99$ in (15), then Simpson's rule hardly appears to converge:

$$
S\left(2^{-5}\right)=.039, \quad S\left(2^{-10}\right)=.072, \quad S\left(2^{-15}\right)=.104 .
$$

On the other hand, the constant $C(h)$ in the error term converges rapidly:

$$
\begin{array}{lll}
C\left(2^{-1}\right)=.994438, & C\left(2^{-2}\right)=.994287, \\
C\left(2^{-3}\right)=.994284, & C\left(2^{-4}\right)=.994238, \\
C\left(2^{-5}\right)=.99423596, & C\left(2^{-6}\right)=.99423534
\end{array}
$$

and

$$
C\left(2^{-k}\right)=-.994235131 \text { for } k=10(1) 15
$$

TABLE 2. $f(t)=t^{-1 / 2} \sin \left(t^{-1 / 4}\right)$

\begin{tabular}{lrrrrr}
\hline$k$ & $2^{k} \times T$ & & & & \\
\hline 1 & .8666 & 6 & 1.5951 & 11 & 1.5103 \\
2 & 1.1810 & 7 & 1.5696 & 12 & 1.5211 \\
3 & 1.3948 & 8 & 1.5319 & 13 & 1.5157 \\
4 & 1.5252 & 9 & 1.5034 & 14 & 1.5102 \\
5 & 1.5867 & 10 & 1.4975 & 15 & 1.5164 \\
\hline
\end{tabular}

Table 2 contains results (using the trapezoidal rule) for the integral

$$
I(b)=\int_{0}^{1} t^{-1 / 2} \sin \left(t^{-b}\right) d t
$$

where $b=.25$. Convergence is very slow and is not monotone. No asymptotic formula $E(h) \approx C_{0} h^{\rho}$ is discernible using either of the two tests (16) and (17). On the other hand, for the small value $b=.01$ convergence is monotone at a rate $E(h)=$ $C(h) h^{.49}$ where $C(h)$ is a slowly varying function of $h$ :

$$
C\left(2^{-1}\right)=1.286, \quad C\left(2^{-5}\right)=1.246, \quad C\left(2^{-10}\right)=1.228 .
$$

At the opposite extreme $b=.49$, convergence is very slow

$$
T\left(2^{-5}\right)=.9477, \quad T\left(2^{-10}\right)=.9972, \quad T\left(2^{-15}\right)=1.0232,
$$


convergence is not monotone and no approximate asymptotic error formula is apparent.

Remark. The exact value of the integral $I(b)$ is a bit complicated to compute. A simple change of variables may be used to put (18) in the form

$$
I(b)=b^{-1} \int_{1}^{\infty} u^{-1-1 /(2 b)} \sin u d u=b^{-1} S\left(1,-(2 b)^{-1}\right)
$$

where

$$
S(x, y)=\int_{x}^{\infty} u^{\nu-1} \sin u d u
$$

is Böhmer's generalized Fensel integral (see Bateman Project [12, Volume II]). The series

$$
S(x, y)=\Gamma(y) \sin \left(\frac{y \pi}{2}\right)-\sum_{m=0}^{\infty} \frac{(-1)^{m} x^{2 m+1+y}}{(2 m+1+y)(2 m+1) !}
$$

works fairly well if $y$ is not a negative integer. For example, only a few terms of this series are necessary to compute $I(.49)=1.023006$. When $y$ is a negative integer, one can utilize the expansion

$$
S(x, y)=x^{\nu}\{P(x) \cos x-Q(x) \sin x\}
$$

where

$$
\begin{aligned}
& P(x)=\sum_{m=0}^{m-1}(-1)^{m}(1-\alpha)_{2 m} x^{-2 m-1}+\mathcal{O}\left(|x|^{-2 m-1}\right), \\
& Q(x)=\sum_{m=1}^{m}(-1)^{m}(1-\alpha)_{2 m-1} x^{-2 m}+\mathcal{O}\left(|x|^{-2 m-2}\right),
\end{aligned}
$$

and $(a)_{n}=\Gamma(n+a) / \Gamma(a)$. For example, this expansion and a quadrature yield

$$
I(.25)=4 \int_{1}^{10} u^{-3} \sin u d u+4 S(10,-2)=1.51412 .
$$

Department of Mathematics

Arizona State University

Tempe, Arizona 85281

Lawrence Radiation Laboratory

University of California

Livermore, California 94550

Center for Dynamical Systems

Division of Applied Mathematics

Brown University

Providence, Rhode Island 02912

1. A. SARD, Linear Approximation, Math. Surveys, no. 9, Amer. Math. Soc., Providence, R.I., 1963. MR 28 \#1429.

2. A. H. STROUD, "Estimating quadrature errors for functions with low continuity," SIAM J. Numer. Anal., v. 3, 1966, pp. 420-424. MR 35 \#6368. 
3. P. J. Davis \& P. Rabinowitz, "Ignoring the singularity in approximate integration," J. Soc. Indust. Appl. Math. Ser. B Numer. Anal., v. 2, 1965, pp. 367-383. MR 33 \#3459.

4. P. RABINOWITZ, "Gaussian integration in the presence of a singularity," SIAM J. Numer. Anal., v. 4, 1967, pp. 191-201. MR 35 \#3881.

5. W. GAUTSCHI, "Numerical quadrature in the presence of a singularity," SIAM J. Numer. Anal., v. 4, 1967, pp. 357-362. MR 36 \#1103.

6. L. Fox, "Romberg integration for a class of singular integrands," Comput. J., v. 10, 1967, pp. 87-93. MR 35 \#3878.

7. P. J. Davis \& P. Rabinowitz, Numerical Integration, Blaisdell, Waltham, Mass., 1967. MR 35 \#2482.

8. E. IsAacson \& H. B. Keller, Analysis of Numerical Methods, Wiley, New York, 1966. MR 34 \#924.

9. K. E. ATKInson, "The numerical solution of Fredholm integral equations of the second kind," SIAM J. Numer. Anal., v. 4, 1967, pp. 337-348. MR 36 \#7358.

10. D. G. SCHWEIKERT, Numerical Parametric Integration of Continuous Functions with a Singular Derivative, Proc. Twenty-Third National Conference of A.C.M., Brandon Systems Press, Princeton, N.J., 1968.

11. R. K. MnLleR, "On ignoring the singularity in numerical quadrature," Math. Comp. (Submitted.)

12. A. ERDÉlYI (Editor), Higher Transcendental Functions. Vol. 2, McGraw-Hill, New York, 1953. MR 15, 419. 